\title{
Políticas del conflicto en El duelo, de Joseph Conrad
}

Eduardo Valls Oyarzun

Universidad Complutense de Madrid (España) 



\title{
Políticas del conflicto en El duelo, de Joseph Conrad
}

\section{Politics of conflict in Joseph Conrad's The Duel}

\author{
Eduardo Valls Oyarzun \\ Universidad Complutense de Madrid (España) \\ evallsoy@ucm.es
}

Fecha de recepción: 1 de septiembre de 2019

Fecha de aceptación: 10 de febrero de 2020

\section{Resumen}

El artículo aborda una relectura de la novela breve The Duel (Joseph Conrad, 1908), que se caracteriza como una revisión crítica de las políticas hegemónicas basadas en el conflicto (aquellas que, hoy por hoy, determinan el fenómeno del populismo). Desde un análisis de la principal premisa retórica de la novela, la conceptualización de la guerra como duelo, el autor presenta las premisas ideológicas que explora al texto: la construcción de un enemigo antagónico en un espacio relacional dialéctico; la afirmación de un estado de excepción como eje de dicho espacio; y la dinámica de la resemantización como forma de mantener vivo el conflicto. El texto de Conrad, visto desde la perspectiva del pensamiento de Deleuze, plantea una crítica a estas políticas identitarias, pues revela un espacio de pensamiento ajeno a la dialéctica, y a través del cual se puede desmontar su mecanismo sistémico.

Palabras clave: Joseph Conrad; The Duel; Hegemonía; Populismo; Gilles Deleuze.

\begin{abstract}
The present article tackles a new reading of Joseph Conrad's novella The Duel (1908). The new approach construes the text as a revision of the politics of hegemony based on conflict-which defines present-day populism. After a thorough an analysis of the main rhetorical principle of the novella, the synecdoche warduel, the author outlines the ideological premises the text scrutinizes, to wit, the construction of an antagonist, i.e. an enemy within the limits of a dialectical space, the assertion of a state of exception as the main ground for the conflict, and the dynamics of re-signification as a means to keep the conflict going. Conrad's text, as seen through Deleuze's perspective, ultimately criticizes these politics of identity and in so doing uncovers a new space of thought, outside dialectics, whereby its systemic structure can be deconstructed.
\end{abstract}

Keywords: Joseph Conrad; The Duel; Hegemonía; Populismo; Gilles Deleuze. 


\section{CONSIDERACIONES PRELIMINARES. INTRODUGGIÓN ${ }^{1}$}

Una de las principales preocupaciones que asolan al autor anglo-polaco Joseph Conrad (1857-1924) en la primera década del siglo XX era la escalada militar europea, que eventualmente culminaría en el estallido de la Gran Guerra. En su ensayo "Autocracy and War" (1925, pp. 83-114), Conrad expresa su inquietud por la lenta pero patente decadencia del Imperio ruso - justo tras haber perdido la guerra con Japón- al tiempo que se producía el también lento, pero no menos patente crecimiento militar y político del Imperio alemán. El ensayo, de marcado carácter pesimista, señala al "black abyss which separates a soulless autocracy (...) from the benighted, starved souls of its people" (Conrad, 1925, p. 89) como causa cabal de la disgregación del Estado ruso. Conrad explicita de este modo las consecuencias deletéreas que trae consigo la dislocación entre la voluntad del "pueblo" y la razón de "Estado", y eleva la circunstancia a categoría de lección política y moral: "This is the real object-lesson of this war, its unforgettable information" (Conrad, 1925, p. 89), por encima incluso de las causas económicas concretas que precipitaron el conflicto ruso-japonés (Conrad, 1925, p. 90). Conrad parece hacerse eco así del pensamiento político de Carl von Clausewitz, quien entiende que la unidad moral entre los componentes del Estado y el Estado en sí mismo (2013, p. 37) es condición sine qua non para la victoria en cualquier conflicto armado, acaso también en cualquier acción política de gran calado.

De algún modo, Conrad nunca pierde de vista esta "unidad moral" en sus grandes textos de la primera década del siglo XX. Puede argüirse que novelas como Nostromo (1904), Under Western Eyes (1911) o The Secret Agent (1907) delimitan un statu quo particular donde dicha unidad se da por liquidada, aunque no siempre con el fin de penetrar en las consecuencias políticas o sociales que se derivan de ella. La lectura tradicional a este respecto la ofrece Frederic Jameson en un célebre comentario sobre Nostromo:

On a second level, there are, of course, Conrad's political reflections and attitudes proper; and he makes it possible for the reader to overlook the identification of his positive figures among the locals -the so-called-Blancoswith the aristocratic party, and that of the evil Monteros with the mestizos: the most explicit statement about Monterist politics is its definition as "Caesarism: the imperial rule based on the direct popular vote" (...). But Nostromo is not a political novel in the sense in which it would allow these two political ideals to fight it out on their own terms (...); rather, Conrad's own political attitudes are presupposed, and rhetorically reinforced by ethical and melodramatic markers (the Blancos are good, the Monteristas evil) (Jameson, 2002, p. 260).

1 El presente artículo ha sido producido y financiado en el marco del Grupo de Investigación "Contextos Literarios de la Modernidad" (Ref.: 941542) de la Universidad Complutense de Madrid, dirigido por el Prof. D. Dámaso López García. 
Alude Jameson a un criterio dialéctico para sacar a Nostromo de la categoría de "novela política", por cuanto no hay desarrollo dialógico entre las facciones en liza (los Blancos -debe incluirse en este bando a los Riberistas-, de una parte; los Monteristas, de otra). Además, Jameson identifica el espacio político de Nostromo con la "actitud política de Conrad", que se presupone en el texto de manera estática, inoperante desde un punto de vista dialéctico. Así las cosas, debe entender el lector que Conrad se muestra reacio a abandonar la retórica del melodrama y sus posibles consecuencias reaccionarias (este es, en esencia, uno de los principales argumentos de Jameson), de suerte que el autor anglo-polaco acaba perpetuando un tipo de estética que podría entenderse como reaccionaria.

Ahora bien, la perspectiva ideológica de Jameson no le permite ver un espacio crítico que vuelve sobre la noción de "unidad moral" expuesta en "Autocracy and War", y que sí constituye una enmienda legítima de orden político a su comentario. No halla Jameson causa para indagar en la homogeneidad de las facciones que se enfrentan en la novela, o acaso siquiera en su articulación; pero sí detecta para el caso el vitriólico comentario del narrador sobre la forma en que los "monteristas" unifican su rebelión contra el Estado. Si en Nostromo se censura el "cesarismo" de los monteristas (Conrad, 1904, p. 335) es porque el texto plantea legítimas dudas -dudas que, sin embargo, Jameson no explora- sobre ese modo particular, orgánico y vertical de homogeneizar pueblo, Estado y "César". Estas dudas ponen de manifiesto una retórica de la sospecha (como se verá a continuación) contra movimientos políticos que intentan recomponer la "unidad moral" de Estado y "pueblo" bajo una identidad colectiva definida en espacios de antagonismo y siempre articulada de arriba abajo. El modo político que mejor comprende este llamado "cesarismo" en el marco de una "narrativa unitaria de la masa" (Urbinati, 2014, p. 131), permítaseme adelantar, es un tipo de política del conflicto que puede denominarse, perfectamente, como "populismo" (Taggart, 2019, p. 79; Urbinati, 2014, p. 131).

El texto de Conrad que acaso despliegue con mayor exhaustividad y perspicacia este tipo de sospecha -por causa de su estilo narrativo y su mecánica de la representación- es una de las novelas breves de su colección A Set of Six (1908), concretamente, The Duel ${ }^{2}$. El relato narra el largo enfrentamiento entre dos oficiales de caballería, D'Hubert y Feraud, protagonistas de una serie interminable de duelos llevados a cabo durante las guerras napoleónicas. Compuesto entre dos grandes cimas de la narrativa de Conrad (Nostromo y The Secret Agent), The Duel ha propiciado muchos más comentarios filológicos que hermenéuticos (véase si no el monumental estudio de fuentes preparado por Stape y Peters, 2015); ha sido calificado, tradicionalmente, como texto menor, o una rareza "that touches no

2 Aunque la primera edición de la novela breve apareció en la colección titulada $A$ Set of Six (1908), el texto se ha publicado por separado en no pocas ocasiones. Esto justifica que me refiera a él en cursiva (The Duel) como obra independiente de la colección en que apareció por primera vez. 
very deep emotions" (Baines, 1993, p. 343); se ha reconocido, en cambio, su valor como espacio narrativo que amalgama al "Conrad romántico" y al "Conrad realista" (Stephen Brodsky, 2014, p. 64) interesado en personajes "who find themselves at the mercy of an absurd universe, unable to control or make sense of their existence" (Peters, 2012, p. 36). También se ha integrado en la revisión que Conrad llevó a cabo hacia la figura y la época de Napoleón (Baines, 1993, pp. 342-343; Stephen Brodsky, 2014, p. 64) y hasta ha sido llevada al cine (The Duellists, Ridley Scott, 1977). Con todo, el canon crítico de Conrad tiene aún pendiente una revisión crítica exhaustiva de The Duel.

Valgan estas páginas como dicha revisión. En el contexto de la sospecha contra el modo político del "conflicto", sostengo que The Duel se ha convertido en un texto insoslayable. En las páginas siguientes mostraré que la novela breve articula un tipo de relación identitaria fundado sobre los principios de una narrativa de lo hegemónico, un proceso dinámico de resemantización de la identidad, así como la imposibilidad ideológica de integración de dicha identidad en una comunidad políticamente funcional. Asimismo, exploraré la naturaleza retórica de estos principios, la cual se sostiene, como se verá, en una sinécdoque que vincula las ideas de "duelo" y "guerra", como ya propuso Carl von Clausewitz en su ensayo seminal De la guerra (1832).

El análisis, de orden retórico e ideológico, planteará primero los principios constitutivos de la política del conflicto; después identificará dichos principios en el texto de Conrad, sugiriendo durante el desarrollo del análisis la presunta inexorabilidad de tales principios; en última instancia se cuestionará dicha inexorabilidad y se describirá el horizonte de pensamiento que la novela implica precisamente para superar el marco político del conflicto. De este modo se intenta re-contextualizar el significado global de The Duel y recuperarlo para el discurso crítico contemporáneo.

\section{RETÓRICA BÉLICA. HEGEMONÍA, DIALÉCTICA Y POLÍTICAS DEL CONFLICTO}

The Duel narra la historia de un joven oficial de caballería (del cuerpo de Húsares), el teniente Armand D'Hubert, a quien se envía a arrestar a otro teniente, de distinto regimiento, de nombre Gabriel Feraud, por haberse batido en duelo con un civil esa misma mañana. A pesar de la delicadeza con que D'Hubert ejecuta sus órdenes, Feraud halla causa para retar a D'Hubert, impromptu, a un duelo. D'Hubert y Feraud se baten inmediatamente, y aunque ambos resultan heridos de cierta gravedad, se niegan a dar por acabada la disputa. Desde entonces, y a pesar de no contar con la aquiescencia del mando militar, Feraud y D'Hubert se baten en una sucesión de duelos durante los siguientes dieciséis años. La disputa alcanza grado de leyenda entre los soldados del ejército napoleónico, debido a la incapacidad que ambos demuestran para vencerse mutuamente, así como al misterio que representa la causa original de la disputa. 
Feraud es gascón (vasco-francés), de carácter sanguíneo y pendenciero, fanático de Napoleón. Tras la caída del emperador, queda condenado a libertad vigilada (en las purgas del ejército francés posteriores a 1818), lo cual, sin embargo, no le impedirá retar a su adversario en duelo una última vez. D'Hubert, por su parte, destaca por ser hombre razonable, frío y precavido, aunque no exento de valor, como corresponde a su estirpe aristocrática. Su carácter cuadra mejor con los requisitos del mando militar profesional en el nuevo ejército monárquico (post-imperial) que el de Feraud. Tras el exilio definitivo del emperador, D'Hubert, hastiado de la vida militar, se lanza a formar una familia. Antes de casarse, sin embargo, accede a batirse por última vez en duelo con Feraud. En el último duelo, Feraud pierde la contienda, pero D'Hubert le perdona la vida con una serie de condiciones que permanecen en vigor varios años. Pasado el tiempo, D'Hubert escribe a Feraud para liberarle de tales condiciones. D'Hubert termina admirándose, ante su esposa, de cómo Feraud fue capaz de definir su vida tantos y tantos años.

La trama de The Duel transparenta una narrativa sobre la idea de conflicto. En el fundamento retórico de dicha narrativa subyace una sinécdoque que vincula la guerra, el todo, con el duelo, la parte: "Napoleon I, whose career had the quality of a duel against the whole of Europe, disliked duelling between the officers of his army (...). Nevertheless, a story of duelling, which became a legend in the army, runs through the epic of imperial wars" (Conrad, 2009, p. 333, énfasis añadido). Este sencillo mecanismo de representación permite leer The Duel como un "paradigm of war" (Stephen Brodsky, 2014, p. 63). Este paradigma resulta ser bastante efectivo, pues propicia innumerables espacios de reflexión sobre la naturaleza del conflicto, sus causas, su alcance, su expresión a lo largo del tiempo $\mathrm{y}$, sobre todo, su uso ideológico en general. Stephen Brodsky, en su artículo seminal sobre The Duel (2014, pp. 63-80), identifica la sinécdoque y la explica en un argumento inductivo que construye el análisis del concepto de duelo en términos que caracterizan la idea de guerra. Acierta Stephen Brodksy en su lectura, pero con ello abre también la posibilidad de pensar el texto a través del proceso inverso, esto es, determinando y caracterizando el concepto de guerra a través de la idea de duelo. Ocurre, además, que esta forma de conceptualizar "la guerra" cuenta ya con una sólida tradición crítica. Así, Carl von Clausewitz, en el primer libro de su tratado sobre la guerra, sostiene:

En lugar de enunciar una definición literaria y petulante de la guerra, iré directamente al meollo de la cuestión: el duelo. La guerra no es sino un duelo a mayor escala. La guerra se compone de innumerables duelos, pero es posible formarse un cuadro del conjunto imaginando una pareja de luchadores. Cada uno de ellos intenta por medio de la fuerza física obligar al otro a hacer su voluntad; su objetivo inmediato es derrotar a su oponente, de modo que sea incapaz de continuar oponiendo ninguna resistencia. Por tanto, la guerra es un acto de fuerza destinado a obligar a nuestro enemigo a hacer su voluntad (Clausewtiz, 1999, pp. 5-6) 
Clausewitz concibe la guerra como una suma total de sus partes, un conjunto global de conflictos individuales (o al menos a "menor escala", parafraseando la cita) orientados a la resolución dialéctica de un choque de voluntades. Este choque es de naturaleza política, pues supone la negociación de la voluntad individual en una estructura colectiva, siquiera de tamaño seminal (la comunidad más pequeña debe contar, al menos, con dos unidades individuales, relacionadas entre sí como amigas o enemigas). A pesar del problema de planteamiento que se deriva de hacer pasar por verdad ontológica aquello que no es sino un truco de representación (la sinécdoque) ${ }^{3}$, Clausewitz inicia con su propuesta la línea argumental que le lleva a exponer su célebre definición de guerra:

La guerra es simplemente una continuación de las relaciones políticas con el añadido de otros medios. Utilizamos deliberadamente la expresión "con el añadido de otros medios" porque también queremos dejar claro que la guerra por sí misma no interrumpe las relaciones políticas o las convierte en algo totalmente diferente (Clausewitz, 1999, p. 494; énfasis añadido).

Así es. Tanto la idea de guerra como el concepto de duelo (permítaseme adelantar) implican continuar las relaciones políticas en un plano extra-dialógico, fundando necesariamente una suerte de "estado de excepción", esto es una "anomical (or alogical) zone of suspensión" en la cual "[to] ground [their] reference to the world of life" (Agamben, 2003, p. 60). En otras palabras, la guerra constituye el límite de masa crítica de la razón, a partir del cual la razón como dinámica objetivable de la política colapsa con el fin, paradójico, de poder administrarse a sí misma . De manera análoga, el duelo propicia que los sujetos involucrados "se [enfrenten] consigo [mismos]", esto es, con su condición de sujetos racionales "porque el duelo lo propicia un movimiento de la voluntad que, cuando elude el control de la razón humana, pervierte tanto la voluntad como la razón" (López García, 2018, p. 65); o,

3 El planteamiento de Clausewitz no resuelve cuestiones políticas fundamentales que surgen al pasar de la escala "menor" a la escala "mayor". Uno de estos problemas, acaso el más evidente, es la naturaleza monolítica e incuestionable de las voluntades que pugnan en el duelo. Si la guerra es un conjunto de duelos, con una multiplicidad de combatientes, hay que entender que las voluntades de dichos combatientes se alinean en torno a un mismo deseo, que es, naturalmente, de naturaleza política. Esto supone, ya de entrada, concebir al combatiente en el estrecho horizonte del "andrós politikon", de suerte que ninguno de los deseos vinculados a cualesquier otras dimensiones de la estructura del sujeto halla cabida en dicha concepción. Conviene esta idealización unidimensional del combatiente (del Hombre, habitualmente el varón) para el análisis de Clausewitz, pues elimina engorrosos matices sobre la compleja estructura del sujeto. Ahora bien, igualmente transparenta, también de entrada, una concepción política que casa bien como se verá más abajo, con la idea de hegemonía o "políticas del conflicto".

4 La guerra en este sentido supone un contexto muy atractivo para la crítica del concepto de razón, así como terreno fértil para tratar las relaciones entre razón y pasión (véase, por ejemplo, Scheipers, 2018, p. 26). Carl von Clausewitz no es ajeno a ello, como bien demuestra el número de páginas que dedica a explorar las múltiples aplicaciones de la razón (en forma de táctica y o estrategia) en el contexto de violencia que preside la guerra (Clausewitz, 1999, pp. 71-214). 
también en otras palabras, porque la razón es capaz de negociar las circunstancias que concurren cuando esta entra en suspenso ${ }^{5}$.

El espacio "anómico (o alógico)" que instauran guerra y duelo se constituye en términos absolutos. Surge en la dialéctica de dos voluntades políticas y colapsa al subsumirse la una en la otra. Como quiera además que dichas voluntades configuran la identidad de los sujetos en liza ${ }^{6}$, la constitución del sujeto en el plano político guerra-duelo debe ser, necesariamente, relacional y dialéctica. Esta premisa revela, en última instancia, la dinámica ideológica del planteamiento como "política del conflicto". Conviene detenerse un momento a explicar este extremo.

Conceptualizar la guerra como espacio político (recuérdese, "la continuación de las relaciones políticas con el añadido de otros medios" (Clausewitz, 1999, p. 494)) conforma una de las estrategias retóricas más reconocibles de los discursos llamados populistas:

For populists there is a strong undertone of politics being the continuation of war by other means. What I mean here is that the repudiation of politics by populists mean that when they engage in political activity the tone, tools and metaphors that they adopt can have more in common with war than with the practice of politics (Taggart, 2019, p. 82).

La adopción de la retórica bélica por parte de los discursos populistas funciona en tres sentidos (Taggart, 2019, pp. 82-83). En primer lugar, implica la construcción del sujeto político en sentido relacional: un "nosotros", o la mayoría pura (Urbinati, 2014, p. 131) se opone de forma dialéctica a un "otro"-o "élite corrupta" (Urbinati, 2014, p. 131)-. Este "ellos", a su vez, se erige necesariamente en "enemigo" a través de la dualidad irreconciliable que ya teorizó Carl Schmitt (2005, p. 56) en su momento y que suele percibirse con nítida claridad cuando se trata de detectar la estrategia populista (Taggart, 2019, p. 83). Así las cosas, el sujeto político populista es, por definición, una categoría ideológica aglutinante y, precisamente por ello, destructiva de aquellos matices de la psique que no compartan los intereses alineados con dicha categoría: "Populism treats pluralism of conflicting interests as a show of litigious claims to be overcome by creating a polarized scenario that simplifies social forces and giving the people the chance to immediately take sides" (Urbinati, 2014, p. 131).

En segundo lugar, la retórica bélica pone de manifiesto el espacio irracional en el que debe dirimirse aquello que Carl Schmitt denomina "lo político" (2005, pp. 50-56). La retórica bélica rechaza la negociación entre las partes en el marco del derecho (Taggart, 2019, pp. 83) y sitúa de ese modo la contienda en un espacio de hegemonía que a su vez justifica la puesta en práctica de algún tipo de "estado de

5 La novela también explora los modos en que la razón administra ese "movimiento de la voluntad" irracional y anómico que conduce al duelo (López García, 2018, p. 65).

6 Véase nota 1. 
excepción". Esto se deriva de lo anterior, es decir, a causa de que la definición del sujeto "nosotros" se realiza en relación con el objeto "ellos":

The war metaphor implies that the enemy are very much an enemy in everything. The elite are essentially to be opposed and these must be a complete defeat. For populism, the war trope justifies the suspension of rights, just as might be expected in the situation of states going to war (Taggart, 2019, p. 83).

Por último, si la construcción lógica del sujeto ("nosotros") depende de la naturaleza ideológica del objeto ("ellos") (Laclau y Mouffe, 2014, pp. 120 y ss.), es necesaria la continua aseveración discursiva del citado objeto para poder establecer la identidad de aquel sujeto: "polarization [is the] means for creating a politics of identity" (Urbinati, 2014, p. 134). En este sentido, cabe no perder de vista que el discurso populista exige establecer un "conflicto continuo" (Taggart, 2019, p. 83), esto es, un estado de guerra total y constante que sostenga el equilibrio relacional entre identidades. Este estado relacional, teorizado como "hegemonía" (Laclau y Mouffe, 2014, pp. 120 y ss.) contiene una paradoja insoslayable: el antagonismo que sustenta la definición de los polos no puede resolverse, so pena de que la identidad del sujeto político se disgregue (eliminado o sometido el "ellos", ya no sería posible definir un "nosotros").

Ahora bien, los padres del concepto parecen ser inmunes a esta disfunción del sistema, que nace por cierto de pensarse este en el horizonte de la dialéctica ${ }^{7}$. La forma discursiva que proponen los Laclau, Mouffe o, previamente (aunque con muchos matices y desde aproximaciones muy distintas) Gramsci y Schmitt para superar esta disfunción es establecer un principio corrector que bien podría denominarse "rearticulación" o "resemantización". Dicho principio corrector consistiría en un proceso discursivo por el cual se reconstruye el "ellos" a partir de la aseveración performativa del componente dialéctico objetivo, esto es, del adversario. Considérense, por ejemplo, las palabras de Chantal Mouffe: "The people and the political frontier that defines its adversary are constructed through political struggle, and they are always susceptible to rearticulation through counterhegemonic interventions" (2018, p. 63). La imposibilidad de renunciar al horizonte de la dialéctica (para salvaguardar el sujeto aglutinante populista) lleva a Mouffe a reafirmar la naturaleza dialéctica del propio sistema (aparece ahora la dinámica "hegemonía" vs. "contra-hegemonía") por razón exclusiva de su lenguaje interno. Produce esto una suerte de reivindicación solipsista del propio sistema -por cuanto el sistema intenta demostrarse a sí mismo-que requiere, en última instancia, de un elemento irracional (la fe en la propia dialéctica) para sostener su validez racional.

7 Este es uno de los principios fundamentales del marco hegemónico que propugnan Laclau y Mouffe (2014, p. 79): todas las identidades son relacionales y, por tanto, se articulan en un espacio dialéctico del antagonismo hegemónico. 
Conviene en este punto recapitular la explicación. La conceptualización de la guerra como espacio político (propio de Clausewitz) ha resultado ser muy productiva para la teorización del modo político populista ${ }^{8}$ (Taggart, 2018, p. 82). Esta se ha valido de dicha retórica para construir un tipo de discurso dialéctico que a su vez se sostiene, sobre todo, en tres pilares: a) la polarización antagónica, irreconciliable y deletérea entre el "nosotros" y el "ellos" (el enemigo); b) la traslación del hecho político a un espacio de excepción en el que se dirima la oposición fundada por causa del anterior punto, y que, naturalmente, por definición, debe acabar con el sometimiento del otro; y, en fin, c) la perpetuación de la dialéctica hegemónica (a través de sistemas de rearticulación y resemantización) como política identitaria. En última instancia, los tres pilares se sostienen entre sí al situarse en el horizonte de la dialéctica; pero, naturalmente, no pueden validarse fuera de dicha estructura sistémica. Se adelanta aquí ya, pues, una forma de desmontar la lógica de este discurso: la posibilidad de pensarlo en un horizonte antidialéctico.

Estos tres pilares configuran en buena medida aquello que bien puede denominarse "política (o políticas) del conflicto". En la siguiente sección se identificarán algunas manifestaciones de estas políticas en The Duel, a fin de explorar los modos como se negocian tales políticas en el texto de Conrad. En última instancia, y como ya se ha sugerido, se buscará un horizonte de pensamiento ajeno a la dialéctica con la que re-pensar la teorización de estas políticas. Esta alternativa, permítaseme adelantar, figura en la novela y casa razonablemente bien con el modo antidialéctico de reafirmación de la diferencia que concibe Gilles Deleuze al interpretar el pensamiento de Friedrich Nietzsche.

\section{GUERRA PRIVADA, GUERRA PERPETUA Y RE-SIGNIFICACIÓN}

Una de las ventajas que trae consigo la sinécdoque rectora de The Duel es la fluida homogeneidad que esta imprime al texto. El planteamiento general de la guerra como un conjunto de duelos, y la conceptualización del duelo como "private warfare" o "a war of [one's] own" (Conrad, 2009, pp. 355 y 388) permite articular el relato como un espacio en el que los planos de figuración retórica se confunden significativamente. Los diversos duelos (con sable, a caballo o con pistola) que ejecutan D'Hubert y Feraud puntúan el crecimiento personal y profesional de los protagonistas. La historia militar de Francia durante las guerras imperiales (focalizada en la desintegración del estado napoleónico) contextualiza la pugna privada entre los dos oficiales; $y$, al hacerlo, se constituye en cierto modo como metáfora del destino trágico que pende sobre ellos.

8 Otro tipo de conceptualización que ha dado muchos frutos en la construcción del discurso populista es la identificación entre "política" y "religión" (Taggart, 2018, p. 84). 
Considérese, a este respecto, por ejemplo, el lamento que expresa D'Hubert al enterarse del compromiso de boda que ha adquirido su hermana (Conrad, 2009, p. 367) y cómo dicho lamento transmuta en la pesadumbre que le produce la absurda disputa con Feraud (Conrad, 2009, p. 366). Su condición militar subyace en la causa de que D'Hubert apenas haya tenido relación con su hermana en la vida adulta. Siente D'Hubert un agudo ataque de melancolía, "of the days of his childhood" (Conrad, 2009, p. 366), que, sin embargo, exorciza redactando su testamento, así como "[giving] himself up to the unpleasant reflection" del duelo con Feraud. El ataque de melancolía de D'Hubert se propicia por lo absurdo de la profesión castrense, pero se orienta hacia la experiencia individual, no menos absurda, de su guerra privada con Feraud. Los conceptos de guerra y duelo resultan así intercambiables; se confunden como planos superpuestos de significación, a pesar de estar bien delimitados retóricamente; y, en fin, trabajan de manera sinérgica, en la misma dirección, a fin de subrayar tanto la deshumanización del soldado como el hastío vital que provoca.

Así las cosas, la "escala menor" de acontecimientos (lo privado, lo particular, lo individual, la biografía) se entreteje con la "escala mayor" (lo público, lo general, lo colectivo, la Historia), creando así una estructura significativa que permite pasar de un espacio al otro sin apenas obstáculos. Esta circunstancia ampara que la micropolítica identitaria de la novela pueda leerse como sinécdoque general de la macropolítica histórica. Y es en este contexto donde las políticas del conflicto se vuelven patentes.

En primer lugar, la identidad de D'Hubert y Feraud se construye, lógicamente, por la estructura relacional del duelo. La peripecia vital que define a cada personaje se imbrica de manera sólida en la del otro, hasta el punto en el que resultan prácticamente inseparables. Los caracteres personales propios de cada uno, en este sentido, surgen siempre en relación con la obsesión particular, la monomanía privada que comparten. Así, por ejemplo, Feraud no guarda reparos en canalizar su carácter pendenciero al objeto de avanzar en el escalafón militar, pero no por causa de una ambición personal concreta (y que sería legítima en cualquier caso) sino más bien para no perder oportunidad de enfrentarse con D'Hubert'.

El código de honor que provee de identidad a ambos personajes (el duelo) se sitúa por encima del derecho castrense; apela a un pretérito anómico en el que el caballero era dueño de su propia vida y podía ponerla en riesgo a voluntad como expresión máxima, precisamente, de su potestad sobre ella- (López García, 2018, p. 64). Hay una parte de voluntad individual romántica involucrada en este planteamiento general (López García, 2018, p. 64); pero el sistema que dota de significado tanto a esa parte concreta del individuo, como al resto del sujeto en el espacio del duelo es, en puridad, de naturaleza colectiva. Esto es así porque el honor

9 Un principio de la ley marcial prohíbe a un oficial de rango inferior retar en duelo a otro de rango superior (Conrad, 2009, p. 364). Feraud y D'Hubert solo se baten cuando comparten los mismos galones. 
personal se administra como prestigio colectivo, de suerte que la restitución de la dignidad desafiada se percibe como deber irrenunciable:

Lieutenant D'Hubert broke in earnestly: "Let me entreat you, Colonel, to be satisfied with taking my word of honour that I was put into a damnable position where I had no option; I had no choice whatever, consistent with my dignity as a man and an officer.... After all, Colonel, this fact is the very bottom of this affair. Here you've got it. The rest is mere detail..." (Conrad, 2009, p. 361).

La explicación del teniente D'Hubert sirve para reafirmar el contexto anómico del duelo (D'Hubert debe atender las obligaciones que le impone el duelo en sí, independientemente de las ordenanzas castrenses), y de paso también para caracterizar su naturaleza antagónica y dialéctica. D’Hubert se erige en enemigo de Feraud (y viceversa) no tanto por causa "del detalle particular" que haya originado la disputa, cuanto por el sistema de deberes y responsabilidades, la articulación de la dinámica dialéctica que genera su identidad. Es en el juego de antagonismos, en la dinámica del desagravio, parece decir D'Hubert, y no en el agravio en sí donde nace el sistema que dota de significado identitario al sujeto. Se pone en práctica pues aquí una identidad relacional, una oposición antagónica polarizada (obsérvese que el duelo, un juego de suma cero, no admite la integración de ninguno de los polos en el otro) cuya resolución por razón de la muerte de uno conllevaría la anulación identitaria del otro. En este sentido, el lector apenas si evita sospechar que el conflicto entre los dos personajes no puede llegar a resolverse jamás.

En efecto, lo verdaderamente novedoso que plantea la novela no resulta tanto el duelo en sí cuanto la absurda manera de no resolverse jamás. Ambos contendientes "refused to have the combat stopped, time after time, with what appeared the most deadly animosity" (Conrad, 2009, p. 363), debido a que sus diferencias particulares "could only be settled by one of the parties remaining lifeless on the ground" (Conrad, 2009, p. 363). Sin embargo, la escrupulosa aplicación de la lógica que administra el espacio irracional del duelo ${ }^{10}$ en su concepción más absoluta e innegociable se encarga de perpetuar el pleito. Esta aplicación depende en exclusiva de los enemigos, aunque estos la hayan articulado en la misma política del conflicto que sostiene el discurso de su "yo". Uno de los duelos más tensos, por el miedo que infunde en D’Hubert, por ejemplo, se despacha así:

And therefore he was more than surprised when, at the very first set-to, Captain Feraud laid himself open to a cut over the forehead, which, blinding him with blood, ended the combat almost before it had fairly begun. It was impossible to go on. Captain D'Hubert, leaving his enemy swearing horribly (...). (Conrad, 2009, p. 368).

10 Recuérdese, la razón como dinámica objetivable de la política colapsa al fin, paradójico, de poder administrarse a sí misma. Véase, más arriba, nota 3. 
"Era imposible continuar", señala el narrador, en forma impersonal, como si se tratara de una circunstancia excepcional, ajena al control de los contendientes. Hay aquí una trampa retórica, naturalmente. Es voluntad de los adversarios llevar a cabo una suerte de "duelo total", de modo que cualquier resultado distinto de la aniquilación del enemigo ha de entenderse como nulo o impracticable. Y como la voluntad es propia, puramente, de los dos sujetos involucrados en el conflicto, es razonable pensar entonces que tal decisión nace en la forma de comprender tanto el propio conflicto como el "yo" que este articula. En efecto, como ya se ha sugerido, no hay forma de sostener el "yo" concebido en el horizonte del conflicto si no es, precisamente, con la perpetuación del conflicto en sí (la identidad entraría en crisis en el momento en que el conflicto se resolviera). Va por tanto en el interés de los dos adversarios el mantener vivo el conflicto, sea al precio retórico que sea.

Ahora bien, Feraud y D'Hubert muestran actitudes distintas ante la forma en que el duelo determina sus identidades antagónicas. Feraud es el personaje más mercurial de los dos. Es Feraud quien activa la política del conflicto (la sucesión de duelos) desafiando a D'Hubert, poniéndole en una "damnable position where I had no option" (Conrad, 2009, p. 361). La ofensa que imagina Feraud para proponer tal desafío es trivial (su detención pública en el salón de Madame de Lionne), pero resulta reveladora del carácter del personaje porque apela a un "objeto de amor propio" (Stephen Brodsky, 2014, p. 68), una expresión solipsista del deseo por medio del cual se revela la condición aislada del propio sujeto al inicio del relato.

Pero Feraud halla en el duelo una dinámica rectora de sus propias acciones. La singularidad del propósito de acabar con D'Hubert define a partir de entonces, casi de forma automática, todos los componentes significativos de su identidad. El antagonismo es tal que Feraud interpreta cualquier acción que cometa D'Hubert como una afrenta personal directa e inexcusable: "I see that I must get rid of that intriguing personage. Here he's managed to sneak on to the personal staff of the marshal. It's a direct provocation to me. I can't tolerate a situation in which I am exposed any day to receive an order through him" (Conrad, 2009, pp. 366). Nótese que en este punto de la historia el pretendido ataque contra la dignidad masculina de Feraud ya no tiene cabida en su discurso. Es la dinámica antagónica lo que subyace a la significación de la ofensa, y no la ofensa en sí, razón por la cual Feraud pierde de vista la afrenta original para ir hallando, puntualmente, distintos motivos con los que perpetuar el duelo.

Hay incluso una gradación en las excusas con las que Feraud mantiene vivo el conflicto: en un principio, como se ha visto, la afrenta ataca la dignidad del amor propio; pero rápidamente el oficial gascón encuentra motivos políticos de mayor calado con los que justificarse:

But it exasperated the higher strung nature of Colonel Feraud. Passing through Magdeburg on service, this last allowed himself, while seated gloomily at dinner with 
the Commandant de Place, to say of his life-long adversary: "This man does not love the Emperor," and his words were received by the other guests in profound silence. (...) Feraud, troubled in his conscience at the atrocity of the aspersion, felt the need to back it up by a good argument. "I ought to know him," he cried, adding some oaths. "One studies one's adversary. I have met him on the ground half a dozen times, as all the army knows. What more do you want? If that isn't opportunity enough for any fool to size up his man, may the devil take me if I can tell what is." And he looked around the table, obstinate and somber (Conrad, 2009, p. 375).

Las palabras de Feraud sugieren que la afrenta de D'Hubert (su falta de amor por el emperador) es posterior a la afrenta que propició el antagonismo entre ellos. Pero el discurso calará posteriormente en círculos bonapartistas, especialmente tras la derrota de Napoleón, hasta que el enunciado "the man who [never loved] the Emperor" (Conrad, 2009, pp. 375, 380, 386, 399 y 414) termina justificando, a ojos de Feraud, el conflicto entre los duelistas. Y esto no es un asunto menor. Feraud logra con este enunciado re-codificar el duelo (la política antagónica del conflicto) en un plano de opresión heterónoma: la clásica oposición discursiva del populismo, "the power versus the underdog" (Finchelstein, 2017, 141). En este caso, los bonapartistas se han convertido, dieciséis años después de que se iniciara la contienda privada entre Feraud y D'Hubert, en marginados del Estado, en un colectivo político desamparado y hasta perseguido por el régimen monárquico; Feraud, sinécdoque individual de la facción bonapartista, denuncia a D'Hubert como sujeto corrupto y traidor, "a sort of monster essentially worse than a mere betrayer" (Conrad, 2009, p. 375). Este constructo político de naturaleza populista, revelado a través de la política del conflicto que rige el relato, es el que articula la crítica principal de Conrad hacia Napoleón y su legado político en el continente, esencialmente un populismo avant la lettre. Así, en "Autocracy and War":

The degradation of the ideas of freedom and justice at the root of the French Revolution is made manifest in the person of its heir; a personality without law or faith, whom it has been the fashion to represent as an eagle, but who was, in truth, more like a sort of vulture preying upon the body of a Europe which did, indeed, for some dozen of years, very much resemble a corpse. The subtle and manifold influence for evil of the Napoleonic episode as a school of violence, as a sower of national hatreds. As the direct provocator [sic] of obscurantism and reaction, of political tyranny and injustice, cannot well be exaggerated (Conrad, 1925, p. 86).

Caracteriza Conrad el régimen bonapartista como cesarismo reaccionario, la degeneración de los principios de la revolución francesa por razón del movimiento autárquico que lo canaliza. Este tipo de movimientos, articulados en torno a políticas identitarias del conflicto (obsérvese como en el pasaje se tilda a Napoleón de "sower of national hatreds") tienen tendencia a acabar, como detecta Conrad, en una "monoanarchy (...)", "a mass unification of the masses under an organic narrative and a charismatic of Caesarist leader personating it" (Urbinati, 2014, pp. 134 y 131). En 
The Duel, Feraud encarna la figura "cesarista"; el oficial gascón aglutina las voces dispersas de la "canaille" (Conrad, 2009, p. 398) que otrora siguieron al emperador y que ahora vagan como espectros de una pesadilla militar y política de la que jamás quisieron despertar. En boca de la aristocracia monárquica francesa, encarnada en el padre de la prometida de D'Hubert, la idea suena así: “Those people don't exist-all these Ferauds. Feraud! What is Feraud? A va-nu-pieds disguised into a general by a Corsican adventurer masquerading as an emperor" (Conrad, 2009, p. 398). Feraud concentra aquí la carga crítica que suscita el principio rector del relato (recuérdese: la sinécdoque entre guerra y duelo), así como sus repercusiones políticas en el propio contexto histórico de la novela. Y lo logra llevando el conflicto con D'Hubert a un plano de significación política superior, donde la apostilla "he never loved the emperor" se vuelve credo privado de la grotesca canaille bonapartista ${ }^{11}$. En el camino, Feraud logra también perpetuar el sistema dialéctico de su conflicto privado, rearticulándolo ahora en torno a la pretendida traición de D'Hubert ("he never loved the emperor") y en cierto modo olvidando la ridícula ofensa personal que lo propició en origen. Este proceso de re-significación, como se explicó en el apartado anterior, resulta ser crítico para articular las políticas del conflicto.

\section{POLÍTICAS DE LA DIFERENCIA. EL ESPACIO ANTIDIALÉCTICO}

Armand D’Hubert, de carácter más sensato, racional y atemperado que Gabriel Feraud, mantiene por lo general una actitud distinta de la de su adversario en relación con el duelo. Tendente a la melancolía, D'Hubert reconoce verdaderamente que "[that] affair had hopelessly and unreasonably complicated his existence" (Conrad, 2009, p. 367); e incluso llega a albergar el presentimiento de que su adversario será el encargado de poner fin a esa misma existencia (Conrad, 2009, p. 365). D'Hubert no halla, pero tampoco busca excusa ni razón para liberarse del compromiso de honor que supone batirse en duelo con Feraud. Pero alcanzado cierto grado de hastío, D’Hubert sí termina poniendo en duda los principios dialécticos que sustentan su identidad como duelista-enemigo de Feraud. Merece la pena examinar en detalle el modo como D'Hubert logra desactivar la política del conflicto que Feraud, por su parte, abraza sin ambages hasta el final de la novela; y merece la pena porque el análisis arrojará luz suficiente para entender cómo pueden desmontarse este tipo de políticas.

El momento de mayor angustia que siente D'Hubert por lo absurdo de su pleito con Feraud ocurre la víspera del duelo que celebran en Silesia. Esa noche

11 El comentario sobre D’Hubert se vuelve ubicuo en la segunda parte de la novela (capítulos III y IV). El propio D'Hubert llega a escucharlo de manera casual en boca de un desconocido que cree a pies juntillas las palabras de Feraud: “"Much good may it do to him," mumbled the elder. "They were both brave men. I never saw this D'Huber-a sort of intriguing dandy, I am told. But I can well believe what I've heard Feraud say of him-that he never loved the Emperor." (Conrad, 2009, p. 380). 
sufre D'Hubert aquel ataque de melancolía donde reflexionaba sobre la profesión castrense y su guerra privada con Feraud, llegando incluso a redactar el comienzo de su testamento y última voluntad (Conrad, 2009, p. 367). Cuando el duelo se declara nulo a la mañana siguiente, D'Hubert vuelve a sus aposentos cegado de júbilo. Una vez allí, destruye la plana de su testamento y escribe a su hermana en los términos siguientes:

"But if you, in the depths of your province," he continued, "ever hear it said that your brother is of a quarrelsome disposition, don't you believe it on any account. There is no saying what gossip from the army may reach your innocent ears. Whatever you hear you may rest assured that your ever-loving brother is not a duellist." (...) He didn't care a snap for what that lunatic could do. He had suddenly acquired the conviction that his adversary was utterly powerless to affect his life in any sort of way; except, perhaps, in the way of putting a special excitement into the delightful, gay intervals between the campaigns (Conrad, 2009, p. 369).

D'Hubert parece haber sufrido una suerte de epifanía. Reniega entonces de su condición de duelista (su identidad mediada por Feraud) al comprender la perversión del sistema dialéctico sobre el que ha sostenido su identidad hasta entonces. D'Hubert parece identificar así la clave de este tipo de estructuras (las políticas del conflicto), y en consecuencia atisba, siquiera aún de manera incipiente, una alternativa práctica a ellas.

La clave de este microsistema político identitario radica en que se trata de una identidad débil, dependiente del objeto relacional contra el que se define; es decir, una identidad "esclava" -como la denominaría Nietzsche (2016, pp. 460-469)subordinada a un elemento externo y ajeno al "yo". Paradójicamente, el sistema político del conflicto se articula para aglutinar la mayor cantidad de poder (Urbinati, 2014, p. 131), cuando realmente lo que hace es colocar la fuente de dicho poder en el objeto antagónico que lo define. Así las cosas, si la identidad del sujeto depende de la negación del objeto relacionado, el "yo" de la parte, o el "nosotros" del todo habrá de definirse como aquello que no es el "ellos" externo; a este respecto, entonces, ese mismo "ellos" solo tiene que alterar su identidad a conveniencia para que el "nosotros" se vea obligado a hacer lo propio.

Se podrá argüir, entonces, que el objeto carece de capacidad para mudar su identidad, puesto que este también se define dentro del espacio dialéctico, es decir, por razón de una interdependencia infinita entre sujeto y objeto. D'Hubert piensa que no. Y Giles Deleuze, piensa que tampoco, pues comprende que la identidad afirmativa no puede, ni debe circunscribirse a un horizonte exclusivamente dialéctico. Propone Deleuze pensar la identidad en un espacio más allá de la dialéctica en aras de articular discursos mediante los que sea posible algún tipo de conceptualización afirmativa. Ese espacio se encuentra en el método de creación de valores que analiza Nietzsche en La genealogía de la moral (1887). El filósofo alemán distingue en su 
libro dos tipos de actividad valoradora: una la ponen en práctica los "señores"; la otra, los "esclavos". Para Nietzsche, el "señor" configura los valores morales desde su propia voluntad, conceptualizando como significado moral sus propios afectos; $y$, al hacerlo, en el fondo acepta una responsabilidad para consigo mismo. "El "señor" descuella así como texto que se escribe a sí mismo, haciéndose él mismo responsable de la relación que él mismo también pueda mantener con el mundo" (Valls Oyarzun, 2017, pp. 233). El "esclavo", por su parte, configura sus valores como derivada de aquellos que ha establecido el "señor", de modo que un cambio de valores por parte de este habría de suponer, también, una alteración del sistema de valores de aquel (Valls Oyarzun, 2017, pp. 233). El "esclavo" coloca pues la responsabilidad de su identidad en el otro (el "señor"), rehuyendo una buena parte de poder y control sobre su propia identidad. Deleuze se pregunta a este respecto, si la relación entre "señor" y "esclavo" funciona en el eje de la dialéctica. Concluye lo siguiente:

¿Quién es dialéctico [en la relación señor-esclavo]? Es el esclavo, el punto de vista del esclavo. El célebre punto de vista dialéctico de la relación señor-esclavo, en efecto, depende de esto: que aquí el poder es concebido, no como voluntad de poder, sino como representación del poder, como representación de la superioridad, como reconocimiento por "uno" de la superioridad del "otro". (...) Es el esclavo quien sólo concibe el poder como objeto de reconocimiento, materia de una representación, baza de una competición, y por consiguiente quien lo hace depender, al final de un combate, de una simple atribución de valores establecidos (Deleuze, 2002, p. 19)

Para desmontar este truco de representación identitario, Deleuze denuncia el antagonismo, es decir, la "oposición y [la] contradicción" (Deleuze, 2002, p. 272) como falsa dinámica relacional, porque, al igual que "el ojo de buey", la dialéctica "refleja una imagen invertida de la diferencia" (Deleuze, 2002, p. 272). Entiende Deleuze que la dialéctica se vale de la diferencia para negarla y, de facto, sustituirla por el principio de "oposición y contradicción". El concepto de diferencia que Deleuze ve en la dialéctica es, en efecto, sesgado. La dialéctica no comprende mecanismos ajenos a la dinámica relacional de la oposición, $\mathrm{y}$, por tanto, solo puede comprender de forma parcial aquello que difiere, pero no la relación orgánica de la diferencia en sí: "La dialéctica hegeliana consiste en la reflexión sobre la diferencia, pero invierte la imagen. Sustituye la afirmación de la diferencia como tal por la negación de lo que difiere; la afirmación de sí mismo por la negación de otro" (Deleuze, 2002, p. 272); La dialéctica y la idea de oposición cancelan el sentido de la diferencia como proceso, o mejor dicho, lo sustituyen por un antagonismo deletéreo aplicado a los polos que difieren entre sí. Por si fuera poco, Deleuze ve un interés heterónomo -en este sentido vale decir "político"- escondido tras este método de pensamiento.

Pero esta inversión no tendría sentido, si no estuviera prácticamente animada por fuerzas que tienen interés en hacerlo. La dialéctica expresa todas las combinaciones de las fuerzas reactivas y del nihilismo, la historia o la evolución de sus relaciones. 
Al ocupar la oposición el lugar de la diferencia, se produce el triunfo de las fuerzas reactivas que hallan en la voluntad de la nada el principio que les corresponde. (...) Finalmente, es el pensamiento del esclavo, expresando la vida reactiva en sí misma y el devenir-reactivo del universo (Deleuze, 2002, p. 273).

Léase de nuevo, a la luz de estos principios, la epifanía de D’Hubert: “He had suddenly acquired the conviction that his adversary was utterly powerless to affect his life in any sort of way" (Conrad, 2009, p. 369). D'Hubert parece darse cuenta de que la política del conflicto en el duelo genera un tipo de identidad según la cual "[man loses] possession of himself" (Stephen Brodsky, 2014, p. 68), al poner el significado de la identidad en la dinámica absoluta de la oposición. La negativa de D'Hubert a ceder el poder de su identidad a Feraud constituye en sí mismo un rechazo a la dialéctica, a los principios de oposición, contradicción y antagonismo como espacio de significación política.

A partir de este punto en el relato, el nuevo D'Hubert tratará de retomar el control de sí mismo, de su propia condición. En cierto modo, el matrimonio de su hermana y la predisposición a entablar una relación sentimental por sí mismo (Conrad, 2009, pp. 389-390) suponen una ampliación conceptual de su identidad; pero sobre todo suponen integrar en la identidad una multiplicidad de significados que surgen por y desde la afirmación de la diferencia, no desde la mecánica de la oposición.

El final de la novela constata precisamente el desmontaje de las políticas del conflicto por parte de D'Hubert. El último duelo del relato recuerda a los lectores la sinécdoque fundamental con la que se urde el texto. D'Hubert propone: "Then let us go into the wood and shoot at sight, while [the seconds] remain outside. We did not come here for ceremonies, but for war-war to the death" (Conrad, 2009, p. 401). Tras rápida escaramuza, Feraud yerra sus disparos contra D'Hubert y este coge desprevenido a su adversario. Pero no lo mata. En su lugar, dicta la siguiente resolución, que para Feraud adquiere rango de ley:

You've forced me on a point of honour to keep my life at your disposal, as it were, for fifteen years. Very well. Now that the matter is decided to my advantage, I am going to do what I like with your life on the same principle. You shall keep it at my disposal as long as I choose. Neither more nor less. You are on your honour till I say the word."

"I am! But, sacrebleu! This is an absurd position for a General of the Empire to be placed in!" cried General Feraud, in accents of profound and dismayed conviction. "It amounts to sitting all the rest of my life with a loaded pistol in a drawer waiting for your word. It's-it's idiotic; I shall be an object of-of-derision."

“Absurd?-idiotic? Do you think so?" queried General D'Hubert with sly gravity. "Perhaps. But I don't see how that can be helped (...). I can't really discuss this question with a man who, as far as I am concerned, does not exist." (Conrad, 2009, pp. 407-408). 
D’Hubert alcanza dos objetivos con su decisión. De una parte, cancela el conflicto general y, con ello, cierra la dialéctica identitaria por la que se ha regido él mismo hasta entonces. De otra, integra la identidad de Feraud en el marco nómico que construye con su discurso. La clave de esta lectura se halla, precisamente, en el enunciado que termina la cita. Puede argüirse que el enunciado implica la negación de la identidad de Feraud. Pero si se comprende bien que la identidad de Feraud se ha construido en el horizonte dialéctico del duelo, resulta imposible soslayar que, una vez superado dicho horizonte, ya no cabe construir la identidad en los límites de su espacio. De ese modo, lógicamente, Feraud deja de existir, como deja de existir el conflicto que lo convierte en referente identitario de D'Hubert.

Al final de la novela, D'Hubert volverá a escribir a Feraud a fin de "to give you back in all form your forfeited life" (Conrad, 2009, p. 413). En ese instante, Feraud se muestra incapaz de hallar identidad fuera del espacio del conflicto, y vuelve a reafirmar su idea de que D'Hubert "never loved the emperor" (Conrad, 2009, p. 414). D'Hubert, sin embargo, renuncia a reactivar la política del conflicto:

My dear, I had the right to blow his brains out; but as I didn't, we can't let him starve. He has lost his pension and he is utterly incapable of doing anything in the world for himself. We must take care of him, secretly, to the end of his days. (...) But for his stupid ferocity, it would have taken me years to find you out. It's extraordinary how in one way or another this man has managed to fasten himself on my deeper feelings (Conrad, 2009, p. 414).

D'Hubert trasciende el espacio dialéctico del conflicto, al mismo tiempo que reconoce el vínculo que ha unido a los duelistas durante tantos años. Y precisamente por ello, una vez el sistema ha colapsado, D'Hubert expresa su sentido del deber para con el bienestar de Feraud. Es en el ejercicio de ese deber donde logra integrar a Feraud en un contexto antidialéctico y, por tanto, afirmativo de la diferencia. D'Hubert coloca el origen de su nueva identidad como amante esposo en su relación con Feraud, de suerte que sin el concurso de este no podría haberse constituido el "nosotros" integrador desde el que ahora habla D'Hubert ("we can't let him starve", "we must take care of him"). En dicho espacio discursivo (el "nosotros"), Feraud figura como autor necesario, como causa afirmativa. Previamente, D’Hubert había reconocido, implícitamente al menos, que Feraud ha quedado desprovisto de identidad, y por ello permite que este continúe reafirmando el discurso de confrontación y conflicto como política identitaria (recuérdese que Feraud sigue insistiendo en su "[he] never loved the emperor"). D'Hubert ya no participa de dicho discurso, pero no por ello renuncia a que Feraud siga afirmándolo (de una manera, acaso, grotesca por solipsista). D'Hubert logra este efecto integrador cuidando de Feraud (epítome, todavía, de dicho discurso) en secreto, es decir manteniendo un vínculo que permita a Feraud afirmar su propia política identitaria. Este vínculo se articula por definición desde la diferencia, pues permite la reafirmación del discurso dialéctico, pero sin participar en él, sin subsumirse en él, rechazando su naturaleza como "representación del 
poder, como representación de la superioridad, como reconocimiento por "uno" de la superioridad del "otro" (Deleuze, 2002, p. 19). En este sentido, y en última instancia, cabe concluir aquí que D'Hubert transparenta, por razón tanto de su responsabilidad hacia Feraud y como de su articulación del "nosotros", una identidad antidialéctica, proteica y compleja en la que coexisten sin razón de oposición o antagonismo los dos componentes que han definido su peripecia: el duelo con Feraud y el matrimonio con su esposa.

Como se ha visto, The Duel explora las relaciones políticas sostenidas sobre la idea de conflicto. En la sinécdoque fundamental del duelo (la parte) como expresión de la guerra (el todo), la novela breve consigue vincular un significado político de escala mayor a la peripecia individual de los duelistas. Estos, D'Hubert y Feraud, a causa tanto de su enfrentamiento como de los discursos de la confrontación que este activa (la política como guerra perpetua), inauguran un espacio dialéctico en y por el cual articular su identidad. Esta se construye en el antagonismo fundamental que define dicho espacio, el cual constituye, en puridad, un contexto de excepcionalidad (se sitúa más allá de la norma militar, se define de manera irracional, pero se articula a partir de la razón, como presupone el espacio político de Clausewitz). El espacio de excepcionalidad, y su uso como estrategia política representan los dos elementos constitutivos de las políticas del conflicto (la oposición antagónica, hegemónica y excluyente de las identidades). Feraud comprende bien el mecanismo de esta estrategia y la abraza sin ambages. Para mantener su vigencia en el tiempo, utiliza el tercer elemento definitorio de estas políticas, a saber, la resemantización, la rearticulación y reajuste del mecanismo dialéctico en función de las contingencias que alteran el conflicto. Este debe perpetuarse al objeto de sostener la política identitaria principal y Feraud lo consigue incorporando la causa colectiva del bonapartismo (ya en decadencia) a una disputa que en origen fue exclusivamente personal. D'Hubert, en cambio, mediante la apertura conceptual de su identidad, logra trascender el espacio dialéctico que la define, por lo general, en los primeros estadios de su peripecia. Al hacerlo, D'Hubert descubre al lector una alternativa antidialéctica al planteamiento defendido por Feraud, planteamiento que resulta muy significativo en aras de poder rearticular las políticas del conflicto fuera del plano dialéctico que las sostiene.

En estos términos The Duel debe leerse como una denuncia del estilo retórico y la representación identitaria macropolítica que se impone a la construcción de la subjetividad en la negociación dialéctica de los espacios colectivos. La novela se construye con los mismos trucos de representación que, sugiere el texto, pretenden pasar por construcción de las identidades colectivas (en este caso disfrazándose además de individuales) en un espacio micropolítico, pero como sinécdoque derivada de lo macropolítico. La superposición de ambos planos hace que el relato sea particularmente efectivo en este sentido, porque revela las dinámicas de poder que vinculan ambos espectros (lo "micro" y lo "macro", lo particular y lo general) en un espacio retórico que llega a convertirse en inexorable "fuerza mayor" política para el sujeto individual. Esta lectura hace que la novela breve descuelle de entre 
las obras de Conrad, pues si bien el autor anglo-polaco explora de forma explícita el conflicto deletéreo inherente a las manifestaciones políticas del "cesarismo" en otros textos (Nostromo, "Autocracy and War"), The Duel articula, en toda su complejidad, el mecanismo retórico que de un modo u otro las hace posible.

\section{BIBLIOGRAFÍA}

Agamben, Giorgio (2003). State of Exception. Trad. Kevin Atteil. Chicago and London: The University of Chicago Press.

Baines, Jocelyn (1993 [1960]). Joseph Conrad. A Critical Biography. London: Weidenfield.

Clausewitz, Carl von (2013 [1816-1830]). Sobre la Guerra. Trad. Celer Pawlowksky. Madrid: Tecnos.

Conrad, Joseph (1925 [1905]). Autocracy and War. Notes on Life and Letters. London: John Grant, pp. 83-114.

Conrad, Joseph (1904). Nostromo. London: John Grant.

Conrad, Joseph (2009 [1908]). The Duel. The Nigger of the Narcissus and Other Stories. New York: Penguin, pp. 333-414.

Deleuze, Gilles (2002 [1967]). Nietzsche y la filosofia. Trad. de Carmen Artal. Barcelona: Anagrama.

Finchelstein, Federico (2017). From Fascism to Populism in History. Oakland: University of California Press.

Jameson, Frederic (2002 [1981]). The Political Unconscious. Narrative as a Socially Symbolic Act. London: Routledge.

Laclau, Ernesto and Mouffe, Chantal (2014 [1985]). Hegemony and Socialist Strategy: Towards a Radical Democratic Politics. London and New York: Verso.

Mouffe, Chantal (2018). For a Left Populism. London and New York: Verso.

Nietzsche, Friedrich (2016). La genealogía de la moral. Obra completa, v. 4. Ed. Sánchez Meca. Madrid: Tecnos.

Peters, John G. (2012). Conrad's Literary Response to The First World War. College Literature: A Journal of Critical Literary Studies, vol. 39, n. 4, pp. 34-45.

Scheipers, Sibylle (2018). On Small War: Carl von Clausewitz and People's War. Oxford: Oxford University Press.

Schmitt, Carl (2005 [1932]). El concepto de lo político. Trad. Rafael Agapito. Madrid: Alianza.

Stape, J. H. y John G. Peters (2015). Conrad's “The Duel”: Sources / Texts. Leiden y Boston: Brill Rodopi. 
Stephen Brodsky, G. W. (2014). An Act Cruel and Absurd: Conrad's Romantic Aesthetic and Realist Ethic. Conradiana. 46. n. 1-2, pp. 63-83. DOI: https://doi.org/10.1353/ cnd.2015.0001

Taggart, Paul (2019). Populism and 'unpolitics'. Populism and the Crisis of Democracy. Vol 1. Concepts and Theory. Ed. Gregor Fitzi, Jürgen MAckert y Bryan S. Turner. New York: Routledge, pp. 79-87.

Urbinati, Nadia (2014). Democracy Disfigured. Opinion, Truth and the People. Cambridge, MA: Harvard University Press.

Valls Oyarzun, Eduardo (2017). Dueños del tiempo y del espanto. Madrid: Escolar y Mayo. 
